\title{
Experimental Demonstration of Holographic Demultiplexer using Volume Diffraction Grating Based on Photopolymer
}

\author{
Jun-Won An*, Nam Kim \\ Research institute for Computer Information Communiaction Dept. of Computer \& Communication Eng., \\ Chungbuk National University, Chungbuk 361-763, KOREA \\ Kwon-Yeon Lee \\ Dept. of Electronics Eng., Sunchon National University, Suncheon-si 540-070, KOREA \\ Sang-Keun Gil \\ Dept. of Electronics Eng., Suwon University, Whasung-shi 445-743, KOREA
}

(Received September 17, 2002)

\begin{abstract}
A 42 channel demultiplexer for dense wavelength division multiplexing(WDM) using photopolymer volumetric diffraction grating that has excellent optical properties and low cost capability, has been designed and optically demonstrated. From the experimental results, we have obtained the 3 $\mathrm{dB}$ bandwidth of $0.18 \mathrm{~nm}$, crosstalk suppression of $20 \mathrm{~dB}$ and channel uniformity of $1.6 \mathrm{~dB}$ for a $50 \mathrm{GHz}$ channel spacing.

OCIS codes: $070.0070,060.4230$.
\end{abstract}

\section{INTRODUCTION}

Optical filters with narrow-bandwidth are key components in applications ranging from spectroscopy to optical communications, from lidar to imaging. In optical wavelength-division multiplexing (WDM) systems, optical filters can be used for wavelength multiplexing/demultiplexing and wavelength-sensitive switching. Particularly, wavelength multiplexers (MUXs) and demultiplexers (DEMUXs) are crucial elements of WDM systems that combine and separate signals at different wavelengths. Typical commercially available WDM configurations offer four to eight channels and future systems will need to operate at 16 or more channels per fiber, with all channels squeezed into the narrow spectral window of erbium-doped fiber amplifiers (EDFAs). To reach these challenges, new optical components are necessary that are capable of multiplexing and demultiplexing ever-greater numbers of channels with very minimum loss, crosstalk, and high reliability [1]. There are several methods of implementing an optical wavelength MUXs/DEMUXs. Among these methods, the current choices include dielectric thin film filters (TFFs), arrayed waveguide gratings (AWGs), and fiber Bragg gratings (FBGs), each have its own advantages and disadvantages. Up to now, the TFFs are the most broadly deployed technology for WDM systems with channel spacing from 400 to $200 \mathrm{GHz}$. But, it is unsuitable for dense WDM systems with channel spacing of $50 \mathrm{GHz}$ or less. AWGs have an inherent size advantage and good wavelength resolution of $100 \mathrm{GHz}$ channel spacing, but these schemes typically introduce large polarizationdependent losses and large insertion losses because of the fiber-waveguide coupling. FBGs can provide 50 $\mathrm{GHz}$ resolutions and have the advantage of being assembled entirely from fiber-optic components. These features make them strong candidates for dense WDM systems. The FBGs are fundamentally two-port devices and in practice must be combined with some sort of cascading devices such as an optical circulator or fiber coupler. These cascading devices are typically expensive, introduce significant insertion losses, and have debatable environmental stability. Recently, holographic wavelength demultiplexers using the volume gratings have been shown to produce very effective optical components [2-5].

In this paper, we present the first experimental study on the holographic demultiplexing scheme based on the photopolymer volume grating, in order to ex- 
plore the potential of this new technique in DWDM applications. To verify the scheme, we present a $50 \mathrm{GHz}$-spaced 42-Channel holographic demultiplexer (H-DEMUX).

\section{STRUCTURE AND DESIGN THEORY}

The basic structure of a H-DEMUX based on photopolymer grating is shown in Fig. 1. It consists of collimating lens, VHG, output-focusing lens. Generally the VHG formed in a photopolymer recording material diffracts all wavelengths with high efficiency into a single diffraction order and so it is possible to construct simple and cost-effective devices. In Fig. 1 the collimated multi-wavelength input beam is incident on the VHG at angle $\theta_{i}$, the input beam is diffracted at a different angle related to its wavelength. The outputfocusing lens transforms the angle difference into a spatial one on the output focal plane. So, the $\mathrm{H}$ DEMUX serves as a wavelength DEMUX by putting a linearly spaced fiber permanently fixed in $\mathrm{V}$ grooves.

For the volume transmission grating, the operating bandwidth with respect to the Bragg angle is governed by the Bragg condition from the grating as follows $[6,7]$

$$
\triangle \lambda_{O P}=\lambda^{2} \cos \theta_{i 1} / 2 n L \sin ^{2} \theta_{i 1} .
$$

Where $\theta_{i 1}$ is the input angle inside the material, $L$ is the thickness of the grating, $\lambda$ is the wavelength, and $n$ is the refractive index. To hold a large number of channel, it is desirable to have a more wide and flat $\triangle \lambda_{O P}$. Once the VHG has been fabricated, the design parameters of our H-DEMUX can be determined by using the following equations:

$$
\begin{aligned}
& d=f \triangle \lambda / \Lambda_{g} \\
& \text { (spatial separationbetween adjacent chnnels). } \\
& \triangle \lambda_{B W}=\lambda^{2} / 2 D \sin \theta_{i} \quad(3 \mathrm{~dB} \text { bandwidth). } \\
& W=\lambda / 2 N A \text { (focal spot size). }
\end{aligned}
$$

where $D$ is the diameter of input beam, $f$ is the focal length of the output- focusing lens, $\Delta \lambda$ is the spectral channel spacing, $N A$ is the numerical aperture of the output lens with $N A=D / 2 f, \Lambda_{g}$ is the grating period with $\Lambda_{g}=\lambda_{W} / 2 \sin \theta_{W}, \lambda_{W}$ is the recording wavelength, and $\theta_{W}$ is the half-crossing angle in air between the recording beams. Using the above equations, one can calculate the number of channels within the usable operating bandwidth $\triangle \lambda_{O P}$ of the VHG. However, from these equations we see that, design refinement is necessary since the parameters influence each other.

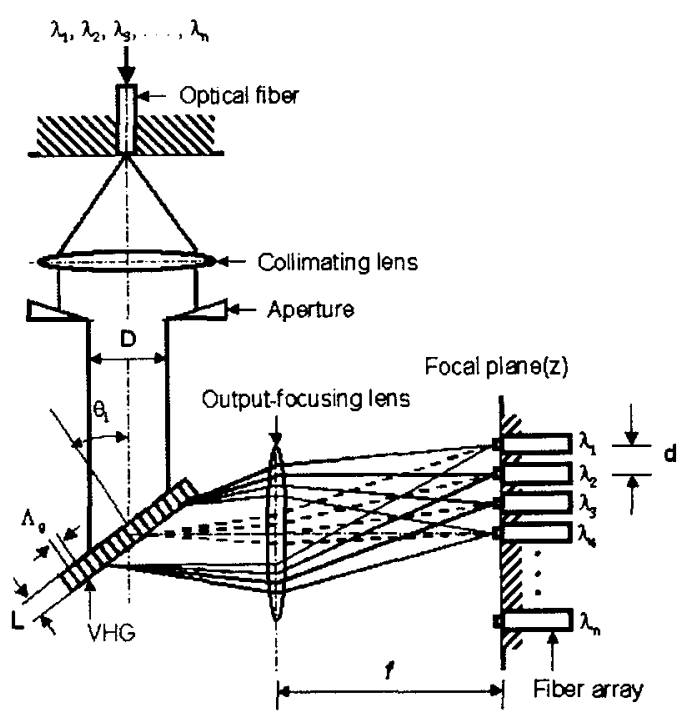

FIG. 1. Scheme of a H-DEMUX based on the photopolymer grating.

In Eq. (3), the $3 \mathrm{~dB}$ bandwidth of each channel with respect to the wavelength is inversely proportional to the diameter $D$. Therefore by increasing the diameter a narrower $3 \mathrm{~dB}$ bandwidth can be achieved. On the focal plane where the light is to be focused onto fibers, it is important to consider those factors that control the coupling efficiency into the fiber. It affects not only the insertion loss but also the crosstalk among channels of the H-DEMUX. For most efficient coupling through direct focusing as shown in Fig. 1, the $N A$ of the lens has to match the $N A$ of the fiber. Moreover, the principal difficulty of this type of device lies in the delicate task of locating and cementing all the fibers in exactly the right place.

\section{EXPERIMENT}

The VHG is made of the Dupont HRF150-38 photopolymer. The $38 \mu \mathrm{m}$ thick film is laminated onto a glass substrate to hold the grating. The optical system for recording the grating provides uniform collimated $1.5 \mathrm{~cm}$ diameter beams derived from a $\mathrm{CW} \mathrm{Nd}-\mathrm{YAG}$ laser at $532 \mathrm{~nm}$. The half-crossing angle $\theta_{W}$ between the recording beams outside the material is $15^{\circ}$, and then the grating period is $1.028 \mu \mathrm{m}$. The performance characteristics of our H-DEMUX are measured using a gain-flattened erbium-doped fiber amplifier (EDFA) source and an Anritsu MS9710B optical spectrum analyzer (OSA). We don't consider the polarization dependence because the readout source has circular polarization. The input beam is collimated by a lens set, and is incident on the grating after passing through the precision calibrated iris diaphragm. The incidence 


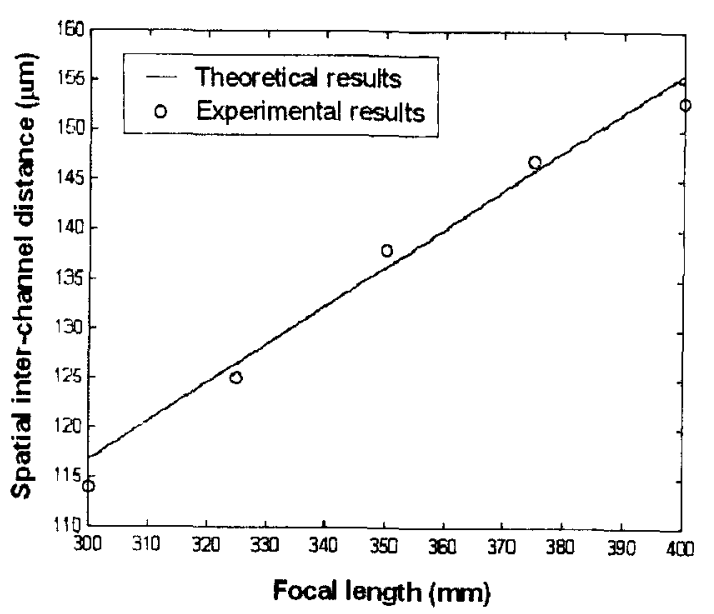

FIG. 2. Spatial inter-channel distance according to the focal length.

angle $\theta_{i}$ at the center-wavelength of $1550 \mathrm{~nm}$ is $48.945^{\circ}$. In these conditions, the $\Delta \lambda_{o p}$ is $72.043 \mathrm{~nm}$. In our configuration to measure the performance characteristics, for experimental convenience we use only a single-mode(SM) fiber in place of a fiber array that is moved and controlled by the motorized fiber alignment unit in the output focal plane. Fig. 2 shows the relationship between the spatial separation and the focal length variation for a given diameter $D=7.5 \mathrm{~mm}$ and incidence angle $\theta_{i}=48.945^{\circ}$ at channel spacing $\triangle=0.8 \mathrm{~nm}$. As expected, the spatial separation is almost proportional to the variation of the focal length. Fig. 3 shows that the $3 \mathrm{~dB}$ bandwidths as a function of the diameter of the input beam. It shows that the theoretical curve agrees very well with experiment, the diameter can be used to tailor the $3 \mathrm{~dB}$ bandwidth response.

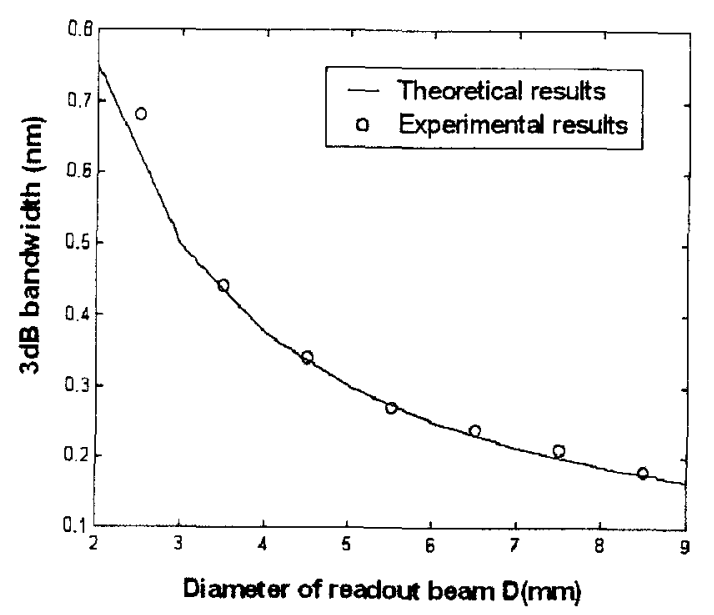

FIG. 3. $3 \mathrm{~dB}$ bandwidth as a function of diameter of readout beam.

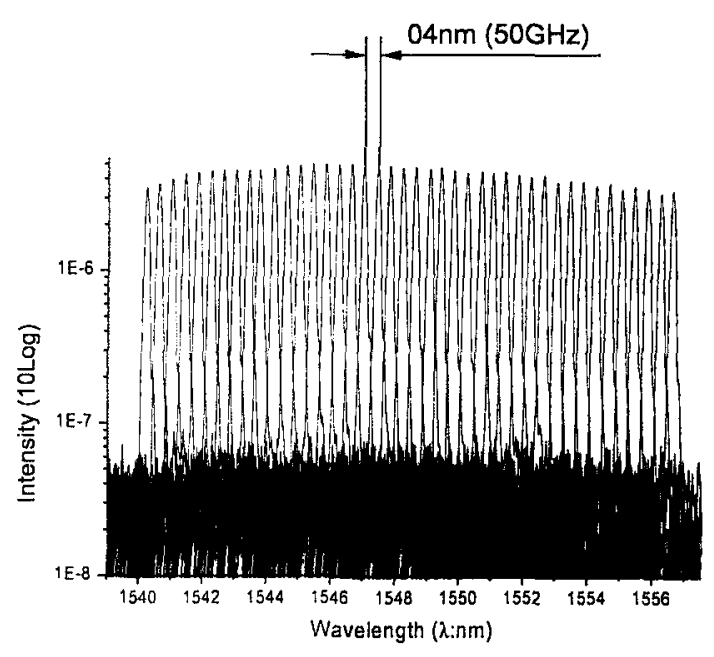

FIG. 4. Spectral response of 42-channel H-DEMUX with $50 \mathrm{GHz}$ channel spacing.

The spectral responses of our 42-channel HDEMUX with $50 \mathrm{GHz}$ channel spacing are shown in Fig. 4. In this test configuration, we chose the focal length of $f=175 \mathrm{~mm}$ and the diameter of $D=8.5 \mathrm{~mm}$. Using the Eq. (2)-(4), the calculated values of the spatial separation $d$, the $3 \mathrm{~dB}$ bandwidth $\triangle \lambda_{B W}$, and the focal spot size $W$ were $69 \mu \mathrm{m}, 0.18$ $\mathrm{nm}$, and $31.911 \mu \mathrm{m}$, respectively.

A SM fiber on the focal plane is moved $69 \mu \mathrm{m}$ each channel, and measured the H-DEMUX spectra. Even if the spot size is wider than the SM fiber diameter, we used the above parameters for the experimental convenience and the simple optical setup. We have measured the channel characteristics in the flat gain region (1540-1557 $\mathrm{nm}$ ) of the EDFA to ensure the power uniformity over the all channels. Experimental result shows that the channel uniformity of about $1.6 \mathrm{~dB}$, the channel crosstalk of $-20 \mathrm{~dB}$, the channel bandwidth of $0.18 \mathrm{~nm}$ and the insertion loss of $8 \mathrm{~dB}$ are achieved. In case of $0.8 \mathrm{~nm}$ channel spacing, the crosstalk of $-28 \mathrm{~dB}$ is obtained. In this experiment, even if the spot size on the focal plane is larger than the SM fiber diameter, we used the above parameters for the experimental convenience and the simple optical setup so that the insertion loss is very high compared to conventional devices. However, it is possible to construct a H-DEMUX component with a lower insertion loss if we have an output-focusing lens that has well matched the NA of the fiber. Moreover, from the Eqs. (2)-(4) we see that the focal length of the output-focusing lens and the diameter of input collimated beam are very critical parameters influencing upon the number of channel $\mathrm{N}$ within the usable operating bandwidth range and the compactness of structure. Thus, by the design refinement and by a proper choice of the components such as lens with a lower F- 
number, VHG with a wider and flatter passband, and fiber array for free space coupling, the overall device performance can be increased considerably.

\section{CONCLUSION}

A 50GHz-spaced 42-channel H-DEMUX based on the VHG formed in photopolymer medium has been demonstrated. In this work, which aims at demonstration the wavelength demultiplex capability of our H-DEMUX, the test configuration used did not adopt the coupling efficiency and the reliable structure of the output port. However, as we have seen, our scheme indeed promises to reduce overall system complexity, and its high spectral resolution (or narrow $3 \mathrm{~dB}$ bandwidth) and low per-channel cost it well to meet the needs of both existing and future DWDM systems.

\section{ACKNOWLEDGEMENT}

This work was supported by grant No.(R01-2001000-00324-0(2002)) from the Korea Science \& Engi- neering Foundation.

*Corresponding author: Jwahn@osp.chungbuk.ac.kr

\section{REFERENCES}

[1] J. R. Bautista, WDM solutions by Laser Focus World, 11 (2000).

[2] V. Leyva, G. A. Rakuljic, and B. O'Conner, Appl. Phys. Lett. 65, 1079 (1994).

[3] R. T. B. James, C. Wah, K. Lizuka, and H. Shimotahira, Appl. Opt. 34, 8230 (1995).

[4] J. W. An, N. Kim, and K. Y. Lee, Elec. Lett. 36, 2092 (2000).

[5] D. Herve, M. Cauvet, J. E. Viallet, and M. J. Chawky, Elec. Lett. 30, 1883 (1994).

[6] S. Breer and K. Buse, Appl. Phys. B, 66, 339 (1998).

[7] J. W. An, N. Kim, and K. Y. Lee, Elec. Lett. 38, 730 (2002). 